\title{
Perfect Matchings in Random Octagonal Chain Graphs
}

\author{
Shouliu Wei $\mathbb{D},{ }^{1}$ Niandong Chen $\mathbb{D}^{2},{ }^{2}$ Xiaoling Ke, ${ }^{1}$ Guoliang Hao, ${ }^{3}$ and Jianwu Huang ${ }^{1}$ \\ ${ }^{1}$ College of Mathematics and Data Science, Minjiang University, Fuzhou, Fujian 350108, China \\ ${ }^{2}$ New Huadu Business School, Minjiang University, Fuzhou, Fujian 350108, China \\ ${ }^{3}$ College of Science, East China University of Technology, Nanchang, Jiangxi 330013, China \\ Correspondence should be addressed to Niandong Chen; 171944938@qq.com
}

Received 13 May 2021; Accepted 14 June 2021; Published 23 June 2021

Academic Editor: Niansheng Tang

Copyright (C) 2021 Shouliu Wei et al. This is an open access article distributed under the Creative Commons Attribution License, which permits unrestricted use, distribution, and reproduction in any medium, provided the original work is properly cited.

A perfect matching of a (molecule) graph $G$ is a set of independent edges covering all vertices in $G$. In this paper, we establish a simple formula for the expected value of the number of perfect matchings in random octagonal chain graphs and present the asymptotic behavior of the expectation.

\section{Introduction}

A general problem of interest in chemistry, physics, and mathematics is the enumeration of perfect matchings, on lattices and (molecule) graphs. Let $G$ be a graph. A perfect matching of $G$ is a set of independent edges covering all vertices in $G$, which is called Kekulé structure in organic chemistry and closed-packed dimer in statistical physics. In organic chemistry, there are strong connections between the number of the Kekulé structures and chemical properties for many molecules such as benzenoid hydrocarbons [1-3]. The number of Kekule structures is an important topological index which had been applied for estimation of the resonant energy and total $\pi$-electron energy $[2,4]$ and Clar aromatic sextet [5]. In crystal physics, the perfect matching problem is closely related to the dimer problem [6-8]. Denote the number of perfect matchings of a graph $G$ by $\Phi(G)$.

An octagonal system (graph) [9] is a finite 2-connected geometric graph in which every interior face is bounded by a regular octagon or quadrangle of side length 1 (see Figure 1). Octagonal graphs have attracted many mathematicians' considerable attentions because of many interest combinatorial subjects. Brunvoll et al. [9] determined the number of isomers of tree-like octagonal graphs by the generating functions. Su and Ding [10] showed that there is a side whose relative length is at most 1 in every convex octagon, and this bound is asymptotically tight. In 2001, Destainville et al. [11] considered some combinatorial properties of fixed-boundary octagonal random tilings. Yang and Zhao [12] presented a relation between the number of perfect matching in octagonal chain graphs and Hosoya index of the caterpillar trees in 2013. Wei et al. [13] discussed the Wiener indices in a type of random octagonal chains in 2018.

An octagonal graph, called an octagonal chain graph, proved that no octagon is adjacent to more than two other octagons. Both the octagonal graphs $G_{2}$ and $G_{3}$ are octagonal chain graphs, as shown in Figure 1. Let $G$ be an octagonal chain graph with $n \geq 3$ octagons labeled by $o_{1}, o_{2}, \ldots, o_{n}$, where $o_{i}$ and $o_{i+1}$ are adjacent for each $i(i=1,2, \ldots, n-1)$. Both the first octagon $o_{1}$ and the last octagon $o_{n}$ are called terminal octagon. And, the remaining octagons $o_{2}, o_{3}, \ldots, o_{n-1}$ are called internal octagon. Each internal octagon is one of type $A$, type $L$, or type $S$ according to whether it separates its two adjacent octagons by a distance of 3, 1, or 2, as shown in Figure 2. A random octagonal chain graph of length $n$ is an octagonal chain graph with $n$ octagons in which each internal octagon is one of type $A$ with probability $p$, type $L$ with probability $q$, or type $L$ with probability $1-p-q$, denoted by $G_{n, p, q}$. Gutman $[14,15]$ studied the perfect matchings about random benzenoid chain graphs in 1990s. Chen and Zhang [16] obtained a simple exact formula for the expected value of the number of perfect matchings in a random phenylene chain. Simple exact formulae are presented for the expected value of the 


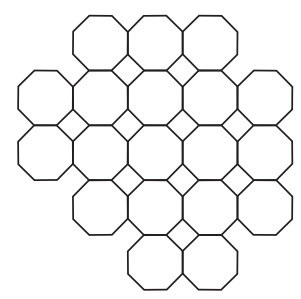

(a)

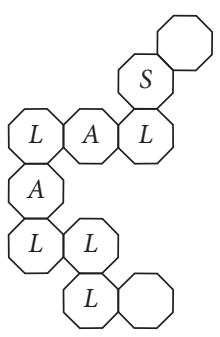

(b)

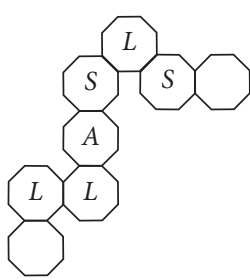

(c)

FIgURE 1: Three octagonal graphs: (a) $G_{1}$, (b) $G_{2}$, and (c) $G_{3}$.

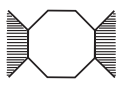

(a)

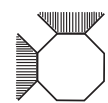

(b)

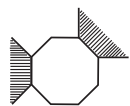

(c)

FIgURE 2: Three types of internal octagons. (a) A. (b) L. (c) S.

number of perfect matchings in random polyomino chain graphs by Wei et al. [17] in 2016. Recently, Wei and Shiu [18] obtained the expected value of the number of perfect matchings in random polyazulenoid chains.

In this paper, we establish an exact formula for the expected value of the number of perfect matchings in a random octagonal chain graph.

\section{The Number of Perfect Matchings in Random Octagonal Chain Graphs}

In this section, we consider the expected value of the number of perfect matchings in a random octagonal chain graph. We will keep the notation defined in Section 1. Recall that there is a recursive formula for the perfect matchings in $G$ [4], i.e.,

$$
\Phi(G)=\Phi(G-u-v)+\Phi(G-u v),
$$

where $u v$ denotes an edge of $G$ incident with the vertices $u$ and $v$. All notations which are not defined in this paper can be found in [19].

Lemma 1. Let $G_{i}$ be an octagonal chain graph with $i$ octagons. Then,

$$
\begin{aligned}
& \Phi\left(G_{1}\right)=2, \\
& \Phi\left(G_{2}\right)=3,
\end{aligned}
$$

and for $i \geq 3$,

$$
\Phi\left(G_{i}\right)= \begin{cases}\Phi\left(G_{i-1}\right)+\Phi\left(G_{i-2}\right), & \text { if the }(i-1)-\text { th octagon is of type } A \text { or } L \\ 2 \Phi\left(G_{i-1}\right)-\Phi\left(G_{i-2}\right), & \text { if the }(i-1)-\text { th octagon is of type } S\end{cases}
$$

Proof. Without loss of generality, let $e=u v$ be an edge in the octagonal chain graph $G_{i}$, as shown in Figures 3 and 4 .

Case 1. Suppose the $(i-1)$ th octagon is of type $A$ or $L$. It is easy to see from Figure 3 that

$$
\begin{aligned}
\Phi\left(G_{i}-u-v\right) & =\Phi\left(G_{i-1}\right), \\
\Phi\left(G_{i}-e\right) & =\Phi\left(G_{i-2}\right) .
\end{aligned}
$$

Thus, we get the result by (1), i.e.,

$$
\Phi\left(G_{i}\right)=\Phi\left(G_{i-1}\right)+\Phi\left(G_{i-2}\right) .
$$

Case 2. Suppose the $(i-1)$ th octagon is of type $S$. It is easy to see from Figure 4 that

$$
\begin{aligned}
\Phi\left(G_{i}-u-v\right) & =\Phi\left(G_{i-1}\right), \\
\Phi\left(G_{i}-e\right) & =\Phi\left(G_{i-1}\right)-\Phi\left(G_{i-2}\right) .
\end{aligned}
$$

Thus, we get the result by (1), i.e.,

$$
\Phi\left(G_{i}\right)=2 \Phi\left(G_{i-1}\right)-\Phi\left(G_{i-2}\right) .
$$

This completes the proof.

Note that the probabilities $p$ and $q$ are unknown constants. Here, $\Phi\left(G_{n, p, q}\right)$ is a random variable. Denote the expected value of $\Phi\left(G_{n, p, q}\right)$ by $E\left(\Phi\left(G_{n, p, q}\right)\right)$.

Lemma 2. Let $G_{i, p, q}$ be a random octagonal chain graph with $i$ octagons. Then,

$$
\begin{aligned}
E\left(\Phi\left(G_{i, p, q}\right)\right)= & (2-p-q) E\left(\Phi\left(G_{i-1, p, q}\right)\right) \\
& +[2(p+q)-1] E\left(\Phi\left(G_{i-2, p, q}\right)\right),
\end{aligned}
$$



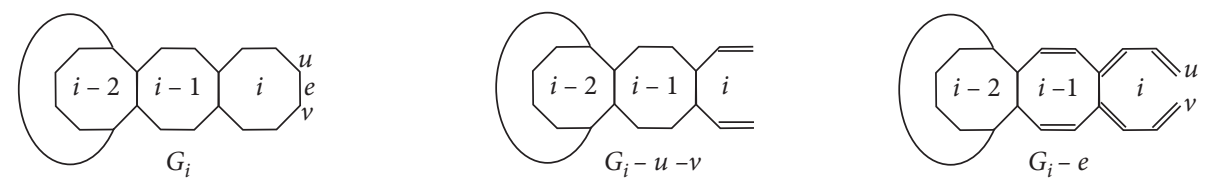

(a)
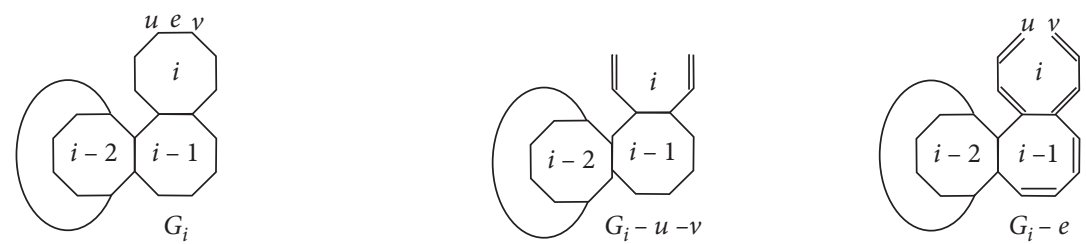

(b)

FIGURE 3: Illustrations of case 1 in Lemma 1: (a) type $A$; (b) type $L$.

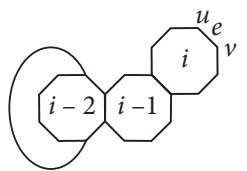

(a)

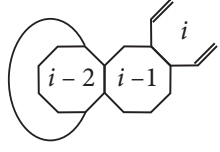

(b)

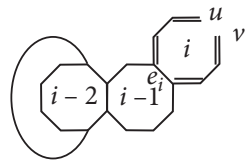

(c)

Figure 4: Illustrations of case 2 in Lemma 1. (a) $G_{i}$. (b) $G_{i}-u-v$. (c) $G_{i}-e$.

where $i \geq 3$.

Proof. Since the $(i-1)$ th octagon of $G_{n, p, q}$ is one of type $A$ with probability $p$, type $L$ with probability $q$, and type $S$ with probability $1-p-q$, we have

$$
\begin{aligned}
E\left(\Phi\left(G_{i, p, q}\right)\right)= & (2-p-q) \Phi\left(G_{i-1, p, q}\right) \\
& +[2(p+q)-1] \Phi\left(G_{i-2, p, q}\right),
\end{aligned}
$$

by Lemma 1. Recall that $E\left[E\left(\Phi\left(G_{i, p, q}\right)\right)\right]=E\left(\Phi\left(G_{i, p, q}\right)\right)$. Since $E\left(\Phi\left(G_{i, p, q}\right)\right)$ is a sum of random variables, we have

$$
\begin{aligned}
E\left(\Phi\left(G_{i, p, q}\right)\right)= & (2-p-q) E\left(\Phi\left(G_{i-1, p, q}\right)\right) \\
& +[2(p+q)-1] E\left(\Phi\left(G_{i-2, p, q}\right)\right) .
\end{aligned}
$$

Thus, the proof is completed.

Theorem 1. Let $G_{i, p, q}$ be a random octagonal chain graph with $i$ octagons.

(1) If $p+q>0$, then, for each $i \geq 2$,

$$
E\left(\Phi\left(G_{i+1, p, q}\right)\right)=\left(\frac{2 r-3}{r-s}\right) s^{i}-\left(\frac{2 s-3}{r-s}\right) r^{i},
$$

where

$$
\begin{aligned}
& r=\frac{2-p-q+\sqrt{(p+q)^{2}+4(p+q)}}{2}, \\
& s=\frac{2-p-q-\sqrt{(p+q)^{2}+4(p+q)}}{2} .
\end{aligned}
$$

(2) If $p+q=0$, then

$$
E\left(\Phi\left(G_{i+1,0,0}\right)\right)=2+i
$$

Proof. Let $e_{i}=E\left(\Phi\left(G_{i+1, p, q}\right)\right)$ and $i \geq 0$. Since $\Phi\left(G_{1}\right)=2$ and $\Phi\left(G_{2}\right)=3$, we have the initial conditions $e_{0}=2, e_{1}=3$. By Lemma 2, we have

$$
e_{i}=(2-p-q) e_{i-1}+[2(p+q)-1] e_{i-2},
$$

for $i \geq 2$. Note that the characteristic equation of (14) is

$$
\lambda^{2}-(2-p-q) \lambda-[2(p+q)-1]=0,
$$

and its characteristic roots are

$$
\begin{aligned}
& \lambda_{1}=\frac{2-p-q+\sqrt{(p+q)^{2}+4(p+q)}}{2} \triangleq r, \\
& \lambda_{2}=\frac{2-p-q-\sqrt{(p+q)^{2}+4(p+q)}}{2} \triangleq s .
\end{aligned}
$$

Case 1. If $p+q>0$, then $r \neq s$. In this case,

$$
e_{i}=k_{1} r^{i}+k_{2} s^{i} .
$$

Substituting the boundary conditions $e_{0}=2$ and $e_{1}=3$, we obtain 


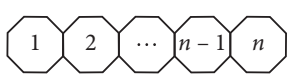

(a)

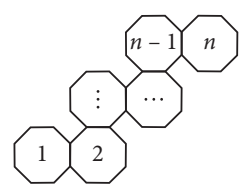

(b)

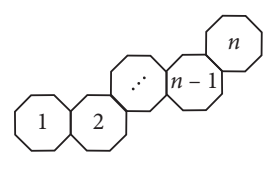

(c)

Figure 5: The parachain $P_{n}$, orthochain $O_{n}$, and metachain $M_{n}$ with $n$ octagons. (a) $P_{n}$. (b) $O_{n}$. (c) $M_{n}$.

$$
\begin{aligned}
& k_{1}=-\frac{2 s-3}{r-s}, \\
& k_{2}=\frac{2 r-3}{r-s} .
\end{aligned}
$$

Therefore,

$$
e_{i}=E\left(\Phi\left(G_{i+1, p, q}\right)\right)=-\frac{2 s-3}{r-s} r^{i}+\frac{2 r-3}{r-s} s^{i}
$$

which proves the first statement of the theorem.

Case 2. If $p+q=0$, then $r=s=1$. Thus, $e_{i}=k_{1}+k_{2} i$, and we have

$$
\begin{aligned}
& k_{1}=2, \\
& k_{2}=1,
\end{aligned}
$$

which means that $e_{i}=E\left(\Phi\left(G_{i+1, p, q}\right)\right)=i+2$.

Let $P_{n}, O_{n}$, and $M_{n}$ be the parachain, orthochain, and metachain with $n$ octagons, as shown in Figure 5 . By assuming $p=1, q=1$, and $p=q=0$, respectively, we can obtain the number of Kekule structures of $P_{n}, O_{n}$, and $M_{n}$ from Theorem 1 .

Corollary 1. Let $P_{n}, O_{n}$, and $M_{n}$ be the parachain, orthochain, and metachain with $n$ octagons. Then,

(1) $\Phi\left(P_{n}\right)=((5+3 \sqrt{5}) / 10)((1+\sqrt{5}) / 2)^{n}+((5-$ $3 \sqrt{5}) / 10)((1-\sqrt{5}) / 2)^{n}$

(2) $\Phi\left(O_{n}\right)=((5+3 \sqrt{5}) / 10)((1+\sqrt{5}) / 2)^{n}+((5-$ $3 \sqrt{5}) / 10)((1-\sqrt{5}) / 2)^{n}$

(3) $\Phi\left(M_{n}\right)=n+1$

It is suggested that the function $E\left[\Phi\left(G_{n, p, q}\right)\right]$ has interest in mathematics and chemistry in [14], especially concerning its asymptotic behavior with respect to $n$. From the explicit expression for $E\left[\Phi\left(G_{n, p, q}\right)\right]$ in Theorem 1, we have the following result.

Corollary 2. Let $G_{n, p, q}$ be a random cyclooctane chain graph with $n$ octagons. If $p+q>0$, then

$$
\lim _{n \longrightarrow \infty} \frac{E\left[\Phi\left(G_{n, p, q}\right)\right]}{E\left[\Phi\left(G_{n-1, p, q}\right)\right]}=r
$$

where

$$
r=\frac{2-p-q+\sqrt{(p+q)^{2}+4(p+q)}}{2}
$$

Proof. If $p+q>0$, then

$$
\begin{aligned}
& r=\frac{2-p-q+\sqrt{(p+q)^{2}+4(p+q)}}{2}, \\
& s=\frac{2-p-q-\sqrt{(p+q)^{2}+4(p+q)}}{2},
\end{aligned}
$$

by Theorem 1. And, by the explicit expression of $E\left[\Phi\left(G_{n, p, q}\right)\right]$, we have

$$
\begin{aligned}
\frac{E\left[\Phi\left(G_{n, p, q}\right)\right]}{E\left[\Phi\left(G_{n-1, p, q}\right)\right]} & =\frac{k_{1} r^{n-1}+k_{2} s^{n-1}}{k_{1} r^{n-2}+k_{2} s^{n-2}} \\
& =\frac{r+s\left(k_{2} / k_{1}\right)(s / r)^{n-2}}{1+\left(k_{2} / k_{1}\right)(s / r)^{n-2}} .
\end{aligned}
$$

Since $r>s$, we obtain

$$
\lim _{n \longrightarrow \infty} \frac{E\left[\Phi\left(G_{n, p, q}\right)\right]}{E\left[\Phi\left(G_{n-1, p, q}\right)\right]}=r .
$$

Thus, the proof is completed.

\section{Data Availability}

No data were used to support this study.

\section{Conflicts of Interest}

The authors declare that they have no potential conflicts of interest.

\section{Acknowledgments}

This work was supported by National Natural Science Foundation of China (no. 12061007), Natural Science Found of Fujian Province (no. 2020J01844), and Science Foundation for the Education Department of Fujian Province (no. JT180392). 


\section{References}

[1] C. M. Grinstead, B. Hahne, and D. Van Stone, "On the queen domination problem," Discrete Mathematics, vol. 86, pp. 2126, 1990.

[2] G. G. Hall, "A graphic model of a class of molecules," International Journal of Mathematical Education in Science and Technology, vol. 4, pp. 233-240, 1973.

[3] R. Swinborne-Sheldrake, W. C. Herndon, and I. Gutman, "Kekulé structures and resonance energies of benzenoid hydrocanbons," Tetrahedron Letters, vol. 16, no. 10, pp. 755-759, 1975.

[4] S. J. Cyvin and I. Gutman, Kekulé Structures in Benzenoid Hydrocarbons, Springer, Berlin, Germany, 1988.

[5] E. Clar, The Aromatic Sextet, Wiley, London, UK, 1972.

[6] P. John, H. Sachs, and H. Zerntic, "Counting perfect matchings in polyominoes with applications to the dimer problem," Zastosowania Matematyki (Applicationes Mathematicae), vol. 19, pp. 465-477, 1987.

[7] P. W. Kasteleyn, "The statistics of dimer on a lattice I, the number of dimer arrangement on a quadratic lattice," Physica, vol. 27, no. 12, pp. 1209-1225, 1961.

[8] M. D. Plummer, "Matching theory-a sampler: from Dénes König to the present," Discrete Mathematics, vol. 100, no. 1-3, pp. 177-219, 1992.

[9] J. Brunvoll, S. J. Cyvin, and B. N. Cyvin, "Enumeration of treelike octagonal systems," Journal of Mathematical Chemistry, vol. 21, pp. 193-196, 1997.

[10] Z. Su and R. Ding, "The relative lengths of sides of convex hexagons and octagons," Journal of Applied Mathematics and Computing, vol. 28, pp. 265-270, 2008.

[11] N. Destainville, R. Mosseri, and F. Bailly, "Fixed-boundary octagonal random tilings: a combinatorial approach," Journal of Statistical Physics, vol. 102, pp. 147-190, 2001.

[12] X. Yang and B. Zhao, "Kekulé Structures of octagonal chains and the Hosoya index of caterpillar trees," Journal of Xinjiang University, vol. 30, pp. 274-277, 2013.

[13] S. Wei, X. Ke, and Y. Wang, "Wiener indices in random cyclooctane chains," Wuhan University Journal of Natural Sciences, vol. 23, no. 6, pp. 498-502, 2018.

[14] I. Gutman, "The number of perfect matchings in a random hexagonal chain," in Graph Theory Notesvol. 16pp. 26-28, New York, NY, USA, 1989.

[15] I. Gutman, J. W. Kennedy, and L. V. Quintas, "Perfect matchings in random hexagonal chain graphs," Journal of Mathematical Chemistry, vol. 6, pp. 377-383, 1991.

[16] A. L. Chen and F. J. Zhang, "Wiener index and perfect matchings in random phenylene chains," MATCH Communications in Mathematical and in Computer Chemistry, vol. 61, pp. 623-630, 2009.

[17] S. Wei, X. Ke, and F. Lin, "Perfect matchings in random polyomino chain graphs," Journal of Mathematical Chemistry, vol. 54, no. 3, pp. 690-697, 2016.

[18] S. Wei and W. Shiu, "The number of perfect matchings in random polyazulenoid chains," Journal of Combinatorial Mathematics and Combinatorial Computing, vol. 105, pp. 21-33, 2018.

[19] J. A. Bondy and U. S. R. Murty, Graph Theory with Applications, Macmillan, New York, NY, USA, 1976. 